\title{
Producción cientifica en salud mental en Perú. Reto en tiempos de reforma de salud
}

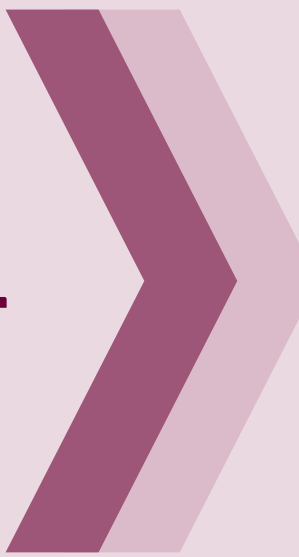

\author{
Scientific production in mental \\ health in Peru. Challenge in \\ times of health care reform
}

\section{Ybeth Luna-Solis ${ }^{1,2}$}

1. Médico psiquiatra, departamento académico de Psiquiatría, Facultad de Medicina, Universidad Nacional Mayor de San Marcos, Lima, Perú.

2. Oficina de Apoyo a la investigación y docencia especializada, Instituto Nacional de Salud Mental Honorio Delgado-Hideyo Noguchi, Lima, Perú.

\section{RESUMEN}

Oвлетіvo. Describir la producción científica en salud mental en el Perú en revistas indizadas en SCOPUS durante el periodo 2011-2013. MateRIAL y MÉtOdos. Se incluyeron artículos publicados en la base de datos SCOPUS, utilizando términos relacionados a la salud mental como: "mental health" or "mental disorders" or "anxiety" or "depresión" or "psycosis" or "psychiatry" or "psychology", dentro del "Title", "Abstract" y "Keywords" de los artículos. Se evaluó la frecuencia de publicación por autor, afiliación de los autores, revista científica de publicación y país. Resultados. Se identificaron 43,135 artículos publicados a nivel mundial entre el 20112013. En el Perú se encontró 26 artículos, que representan el $0.06 \%$ de la producción científica a nivel mundial. Además de ser menor en 16 veces comparado con Brasil, 2.6 con Chile, 1.8 con Argentina y 1.5 con Colombia; sin embargo, mayor respecto a Venezuela, Uruguay, Ecuador, Bolivia y Paraguay. La mayoría de los artículos se encuentran publicados en la Revista Peruana de Medicina Experimental y Salud Pública. Los autores publicaron en promedio entre 1 y 2 veces sobre este tema, afiliados básicamente a instituciones académicas y solo algunos en instituciones de salud. Siendo la mayoría autores extranjeros. Conciusión: En tiempos de cambios y de reforma, fomentadas para el aumento de las prestaciones asistenciales en los temas de Saludy Salud Mental, se observa una escasa atención a la investigación y al desarrollo de la literatura científica sobre Salud Mental, debido a la pobre normatividad sobre ese tema. La producción científica en salud mental en el Perú continúa siendo muy escasa en base de datos importantes como SCOPUS, mientras que mantenemos las diferencias en publicaciones con respecto a Brasil, Chile, Argentina y Colombia. La participación de autores extranjeros es mayoritaria en publicaciones halladas sobre salud mental en el Perú.

Palabras clave: producción científica en salud mental; reforma de Salud; Perú

\begin{abstract}
GOAL: Describe the scientific production in mental health in Peru in journals indexed in SCOPUS during the period 2011-2013. Material AND Methods. Articles published in the SCOPUS database were included, using terms related to mental health as "mental health" or "Mental disorders" or "anxiety" or "depression" or "Psycosis" or "psychiatry" or "psychology" within the "Title", "Abstract" and "Keywords" articles. The frequency of publication by author, affiliation of authors, scientific journal publishing and country were evaluated. Results: 43,135 papers published worldwide during 2011-2013 were found. In Peru 26 papers, representing $0.06 \%$ of the worldwide scientific production was found. In addition to being 16 times lower than Brazil, 2.6 to Chile, 1.8 to Argentina and 1.5 to Colombia; however, higher than Venezuela, Uruguay, Ecuador, Bolivia and Paraguay. Most are published in the Revista Peruana de Medicina Experimental y Salud Pública. The authors have been published between 1 to 2 times on this issue and basically affiliated academic institutions; however also come from institutions of health. As most foreign authors. Conciusion: In times of change and reform, promoted for the increase of welfare benefits in health and Mental health issues, the choice of the location of the research and the development of the literature on Mental Health, is due to the fact that nothing or very little standard on that topic. The scientific production in mental health in the Peru continues to be very low, as found in SCOPUS. Despite the years we maintain differences in publications concerning Brazil, Chile, Argentina and Colombia. The share of foreign authors is majority found in publications on mental health in Peru.
\end{abstract}

KEY WORDS: scientific production in mental health; reform of health; Peru 


\section{INTRODUCCIÓN}

En tiempos de cambios y de reforma, fomentadas para el aumento de las prestaciones asistenciales en los temas de Salud, se encuentra la disyuntiva entre la actividad asistencial y el desarrollo de la literatura científica sobre salud y salud Mental en nuestro país, debido a que nada o muy poco se ha normado sobre ese último tema. Sin embargo, la OMS en su Informe sobre la salud en el mundo 2013: Investigaciones para una cobertura sanitaria universal, indica que para lograr los objetivos de desarrollo del milenio, próximo a concluir en el 2015, se debe trabajar políticas necesarias para el desarrollo de la salud pública basadas en la investigación, condición indispensable para el desarrollo del sistema de salud y la tecnología, logrando prestaciones y acceso a servicios de salud de alta calidad de manera sostenible y local. ${ }^{1}$

Durante el año 2013, se publicaron 23 Decretos Legislativos por el Ministerio de Salud, todas ellas relacionados a las prestaciones asistenciales, encargadas de regular, asistir y/o fortalecer los servicios de salud, sus redes y los recursos que los componen. ${ }^{2}$ Para difundirlas, a través de los medios de comunicación, el Ministerio de Salud emite la RM 791-2013, que con un aporte de casi 18 millones de soles, el país debiera esperanzar el desarrollo de la salud basados en condiciones asistenciales. Los lineamientos y medidas de reforma del sector salud “El Perú Saluda la Vida”, publicados en julio del mismo año, dan cuenta que la política de reforma se orienta a la construcción de un sistema integral de salud para conseguir eficacia y eficiencia en el sector; pero no hace mención, ni aún en las propuestas de mandato de políticas relacionados a la unidades de formación académica tanto de pre y posgrado, sobre la capacidad investigativa en salud ni en salud mental. ${ }^{3}$

La investigación se encuentra estrechamente ligada al avance de la ciencia médica, siendo la metodología científica el eje central para la labor asistencial. ${ }^{4}$ Esta plantea soluciones ante los diversos problemas sanitarios, puesto que sus resultados generan un gran impacto sobre los sistemas de salud, sus políticas y sus prácticas, tanto para la salud individual como colectiva, e incluso son la base científica necesaria para brindar el financiamiento de las intervenciones asistenciales y la toma de decisiones y generación de políticas. $^{5}$

Uno de los indicadores de productividad de las investigaciones, se encuentran en el número de investigaciones que han logrado ser publicadas en revistas indizadas. ${ }^{6}$ Situación difícil de concretar encontrándonos en el puesto 120 en el ranking global de competitividad de 144 países. ${ }^{7}$ Sin embargo, el acceso a la literatura científica ha mantenido relativo avance. En este sentido, el Consejo Nacional de Ciencia, Tecnología e Innovación Tecnológica (Concytec), órgano rector del sistema de investigación en el Perú, contribuyendo a las investigaciones, ha facilitado la accesibilidad a la información científica con nuevas bases de datos, que se encuentran en su página Web (http://bvcyt. concytec.gob.pe/php/index.php). ${ }^{8}$

Scopus es una de las que más resalta. Suele ser utilizada para trabajos de investigación por la gran cantidad de resúmenes y referencias bibliográficas de literatura científica con que cuenta. Adicionalmente, es una herramienta para estudios bibliométricos y evaluaciones de producción científica. ${ }^{8}$

Scopus abarca $50 \%$ del universo de revistas científicas que componen el directorio internacional de publicaciones seriadas Ulrich's, mientras que el Web of Science, otra base de datos bibliográficos, procesa solo $25 \%$ de ellas. También procesa $95 \%$ de las fuentes bibliográficas que ingresan a Web of Science, y $100 \%$ de lo indizado por Medline (que a su vez, comprende más de $90 \%$ del total de registros existentes en PubMed), bases de datos bibliográficas de literatura médica más utilizadas a escala mundial. ${ }^{9}$

Ante los beneficios mencionados se decidió utilizarlo como base de datos referencial para el estudio bibliométrico de esta investigación.

El objetivo del presente artículo es describir la producción científica en salud mental en el Perú en revistas indizadas en Scopus de 2011 a 2013. El periodo comprendido está en relación, a las ya existentes múltiples revisiones sobre publicaciones de literatura científica en salud y en salud mental de nuestro país en bases de datos bibliográficos, entre los años 2001 al 2010. 4,10,11,12,13 


\section{MATERIAL Y MÉTODOS}

Se realizó el análisis de la literatura científica de 2011 a 2013, en la base de datos de revistas biomédicas indizadas de Scopus, para lo cual se utilizó palabras claves relacionados a la salud mental en el "Title", "abstract" y en "keywords", tales como "mental health" or "mental disorders" or "anxiety" or "depresión" or "psycosis" or "psychiatry" or "psychology. Se evaluó la frecuencia de publicación por autor, afiliación de los autores, revista de publicación y país. Se revisó uno a uno las publicaciones halladas eligiendo las que cumplían con el criterio de desarrollo del tema de salud mental, además, en su contenido.

\section{RESULTADOS}

Se han publicado 43,135 artículos a nivel mundial durante el periodo 2011-2013. ${ }^{14}$ En el Perú, encontramos 23 artículos de salud mental, que representa al $0,06 \%$ de la producción científica mundial. En relación a las publicaciones en otros países sudamericanos, Brasil cuenta con 426 artículos, Chile con 68, Argentina con 47 y Colombia con 39. Sin embargo, Perú es mayor que Venezuela con solo 16, Uruguay con 6, Ecuador con 5, Bolivia con 4 y Paraguay con 3 publicaciones. Es la Revista Peruana de Medicina Experimental y Salud Pública la que más ha publicado, con 4 artículos indizadas en Scopus. Pero ha contribuido con 8 artículos más, relacionados con violencia y adicciones, ${ }^{15}$ palabras claves no incluidos en esta búsqueda. Las demás revistas halladas son extranjeras y en cada una se encuentra una sola publicación sobre el tema. La participación de investigadores extranjeros ha sido mayoritaria, representando $56 \%$ (49) del total de investigadores que han publicado durante el periodo de 2011 a 2013. Los autores han publicado entre una y dos veces, y afiliados a instituciones académicas, como la UPCH, seguido del King's College London. Dentro de instituciones privadas de salud tenemos a Psicosalud y Socios en Salud. Las afiliaciones son mayores en instituciones extranjeras, destacando las provenientes de EE. UU. e Inglaterra. ${ }^{14}$ Las afiliaciones en instituciones de salud públicas son bajas. Sin embargo, ubicamos dentro de las más frecuentes al Instituto Nacional de Salud con cuatro afiliaciones, al Hospital Cayetano Heredia y Hospital Hipólito Unanue con tres afiliaciones respectivamente, seguidos del Instituto Nacional Materno Perinatal y el Hospital Dos de Mayo con dos afiliaciones cada una, y del Hospital Hermilio Valdizán, Hospital Alberto Sabogal, Instituto Nacional de Ciencias Neurológicas, Hospital San Bartolomé y al Hospital Edgardo Rebagliati Martins, cada uno con una afiliación.

\section{DISCUSIÓN}

EL estudio muestra un resultado similar a lo publicado en la Revista de Neuro-Psiquiatría, en 2011, donde se encuentra, entre los años 2001 a 2010, que la producción científica sobre salud mental, en nuestro medio, contribuía solo con $0,03 \%$ de las publicaciones a nivel mundial, condición que ha variado ligeramente, a pesar de ser un análisis de tres años, posteriores al estudio inicial. ${ }^{10}$ La literatura científica sigue concentrada en Lima, hallazgo también similar a lo publicado en el 2011. ${ }^{10}$ Sin embargo, en comparación al número de publicaciones por año, en 2011, se indizaron en Scopus 16 artículos; en 2012, 7 artículos y en 2013, solo 3 publicaciones. Esta última situación difiere de lo encontrado en la Revista de NeuroPsiquiatría, que hallaban por año un incremento en el número de publicaciones, así para el 2001 se contaba con 5, mientras que para el 2010 ya se tenía 32 publicaciones. ${ }^{10}$

La participación en publicaciones de instituciones extranjeras en colaboración con instituciones nacionales, ha permanecido elevada durante el período 2011-2013, condición similar a lo hallado en Scopus durante la década pasada. Así mismo, se encontró que gran parte de los autores están afiliados a instituciones académicas más que instituciones laborales.

En comparación con los artículos indizados en Scopus, según países sudamericanos, durante el 2011 al 2013, seguimos con menor producción científica en 16 veces comparado con Brasil, 2.6 con Chile, 1.8 con Argentina y 1.5 con Colombia, aunque mantenemos un mayor número de publicaciones en 8 veces que Paraguay, 6.5 que Bolivia, 5.2 que Ecuador, 4 que Uruguay y 1.6 que Venezuela. Esto podría responder al interés de sus gobiernos y al porcentaje de PBI asignado para los proyectos de investigación y desarrollo $(I+D)$, 
así a nivel de América Latina, Brasil presenta el porcentaje más alto de inversión con un $0.9 \%$ de su PBI, seguido de Chile con $0.7 \%$ y Argentina con $0.6 \%$.

El Perú, se encuentra entre los países que menos invierte en investigación con un $0.15 \%$, estando apenas por encima de Ecuador y Paraguay. Es importante también mencionar que, en los países en vías de desarrollo, es el sector público, el que asume la mayor fuente de financiamiento. ${ }^{16} \mathrm{En}$ este punto, la OMS tiene un rol trascendental en la promoción y ejecución de las investigaciones, como principal organismo sanitario internacional, colaborando con todas aquellas instituciones gubernamentales o no, para investigar y alcanzar beneficios sanitarios a un mayor número de personas. Esto nos da la oportunidad de desarrollar investigaciones que se traducirían en políticas y

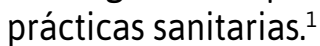

Otra explicación podría centrarse en que el Perú presenta un déficit de investigadores a tiempo completo, siendo estos, según el Ministerio de Economía y Finanzas, de 0,24 investigadores a tiempo completo por cada 1000 participantes en la población económicamente activa; mientras que Chile presenta un ratio de 2 por cada $1000 .{ }^{16}$ En 2004, habían solo 5000 investigadores, $45 \%$ representaban al sector privado (empresas); $40 \%$, a educación superior; $13,9 \%$, al sector público y $0,1 \%$, a organizaciones privadas sin fines de lucro. ${ }^{17}$

Mientras se mantengan limitaciones para la inversión en investigación, un sistema pobre en redes de intervención investigativa y leyes que restringen o no permitan el desarrollo de la investigación, seguiremos con bajos indicadores de producción científica, y temas de salud mental casi inexistentes, a pesar de nuestro despegue económico.

\section{CONCLUSIONES}

Se encuentran escasas publicaciones en revistas indizadas en Scopus, durante el periodo de 2011 a 2013, de las distintas instituciones psiquiátricas y de aquellas que cuentan con servicios de psiquiatría.

En base a la coyuntura de reforma y de énfasis en las prestaciones asistenciales, las dificultades para investigar son mayores y la producción científica en Salud Mental más escasa, hallándose una tendencia menor de publicaciones en cada año del periodo comprendido en el estudio, en comparación a lo publicado en los años de la última década.

La participación de autores extranjeros y predominante hallazgo de afiliaciones en instituciones externas al país, forman parte relevante de lo encontrado. En contraposición, las afiliaciones en instituciones públicas asistenciales de salud son escasas, según los resultados de nuestro estudio.

En el Perú no se ha consolidado una cultura basada en la investigación científica, generando que se prioricen políticas no relacionadas con ella, lo que mantiene una baja producción de literatura científica a nivel mundial y en Latinoamérica. Por otro lado, se observa la necesidad de un mayor financiamiento por capitales privados para la investigación. Es necesario seguir formando capital humano para investigaciones que respondan a las necesidades de salud de nuestro país, con participación activa de las redes de salud y las instituciones académicas, así como otras instancias inmersas en el tema. ${ }^{5}$

\section{REFERENCIAS BIBLIOGRÁFICAS}

I. OMS. Informe sobre la salud en el mundo 2013: Investigaciones para una cobertura sanitaria universal. Genova, Organización Mundial de la Salud; 2013 [citado el 3 de marzo de 2014]. Disponible en: http://apps.who.int/iris/ bitstream/10665/85763/I/9789240691223_spa.pdf

2. Minsa [Internet]. Lima, Perú: Ministerio de Salud; 2014 [citado el 3 de enero de 2014]. Disponible en: http://www.minsa.gob.pe/ portada/Especiales/2013/reforma/decretos.html

3. Minsa. Consejo Nacional. Lineamientos y medidas de reforma del sector Salud. Lima: Minsa; 2013.

4. Gutierrez C, Mayta P.Publicación desde el Pregrado en Latinoamérica: importancia, limitaciones y alternativas de solución. Ciencia e Investigación Médica Estudiantil Latinoamericana. Portal Revistas Peruanas. Cimel. 2003 [citado el 7 de enero de 2014]. Disponible en: http://revistas.concytec.gob.pe/scielo. php?pid=S1680-839820030001000 10 \&script=sci_arttext

5. Cabezas C. Necesidad de la investigación en salud para contribuir a la equidad, la salud y el desarrollo. Rev Peru Me Exp Salud Publica. 2010;27(3):310-II.

6. Rojas-Revoredo V. Las publicaciones en revistas indexadas, único indicador de la producción de las sociedades científicas estudiantiles. Cimel. 2007; I2(I):5-6.

7. Concytec, editores. Crear para crecer. Estrategia nacional para el desarrollo de la ciencia, tecnología e innovación [Documento sujeto a consulta pública]. Lima: Concytec; 2014 [citado el 8 de setiembre de 2014]. Disponible en: http://portal.concytec.gob. pe/images/stories/images2014/mayo/crear_crecer/estrategias_ 


\section{Acta méaitica Permana}

crear_crecer_ultima_version_28-5-2014.pdf

8. Concytec [Internet]. Lima: Consejo Nacional de Ciencia, Tecnología e Innovación tecnológica. Concytec; 2005 [citado el 28 de enero de 2014]. Disponible en: http://portal.concytec. gob.pe/index.php/concytec/areas-de-la-institucion/informacioncomunicacion/informacion-cientifica/literatura-cientifica.html

9. Cañedo R, Rodríguez R, Montejo M. Scopus: la mayor base de datos de literatura científica arbitrada al alcance de los países subdesarrollados. Acimed [Internet]. 2010 [citado el II de febrero de 2014]; 2 I(3): 270-282. Disponible en: http:// scielo.sld.cu/scielo.php?script=sci_arttext\&pid=SI024$94352010000300002 \& \operatorname{lng}=\mathrm{es}$

10. Vega-Dienstmaier J. La literatura científica peruana sobre salud mental. Rev de Neuropsiquiat. 20I I;74(4):285-6.

II. Humaní C, Mayta-Tristán P. Producción científica peruana en medicina y redes de colaboración, análisis del Science citation index 2000-2009. Rev Peru Med Exp Salud Pública. 2010;27(3):315-25.

12. Livia J. La producción científica y los estudios de post grado en Psicología en el Perú. R. Interam Psicol. 2008;42(3):43I-45.

13. Taype-Rondan A, Lajo-Aurazo Y, Huamani C. Producción científica peruana sobre trastornos mentales en Scielo-Perú, 2006-20II. Rev Med Hered [internet]. 2012 Jul [citado el II de febrero de 2014];23(3):I66-I7I. Disponible en: http://www.
scielo.org.pe/scielo.php?script=sci_arttext\&pid=S10 $18-130 \times 20$ I 2000300005\&lng=es\&nrm=iso.

14. Scopus. Search.[Internet]. [citada el I de febrero de 2014]. Disponible en: http://www.scopus.com/search/form.url?zone= TopNavBar\&origin=resultslist

15. Instituto Nacional de Salud [Internet]. Lima= Instituto Nacional de Salud; 2014 [citado el 10 de febrero de 2014] Disponible en: http://www.ins.gob.pe/rpmesp/numeros.asp

16. Bermúdez JE. Investigación científica en el Perú: factor crítico de éxito para el desarrollo del país. Portal de Revistas UPC. 2008;2: I-I7 [citado el II de febrero de 2014]. Disponible en: http:// revistas.upc.edu.pe/index.php/sinergia/article/view/I 30

17. Gestión [internet]. Lima, Perú; invierte solo el $0.15 \%$ de su PBI en ciencia y tecnología, mientras que Chile destina el $0.5 \%$. Gestión; 2014 [citado el 30 de enero de 2014] Disponible en: http://gestion.pe/economia/gobierno-peruano-invierte-solo0I5-su-pbi-ciencia-tecnologia-innovacion-mientras-que-chileinvierte-05-2087516?href=nota_uhora.

\section{Correspondencia}

Ybeth Luna Solis.

ybethluna@hotmail.com

Fecha de recepción: II de enero de 2014

Fecha de aceptación: 13 de febrero de 2015

\section{Conflictos de interés}

El autor declara no tener conflictos de interés durante el planteamiento, ejecución de la investigación y la elaboración del artículo para su publicación. 